Introduction

\title{
Le Genre globalisé : cadres d'actions et mobilisations en débats
}

Globalised Gender : Discussing Frames of Actions and Mobilisations

Delphine Lacombe, Elisabeth Marteu, Anna Jarry-Omarova et Brigitte Frotiée

\section{(2) OpenEdition \\ Journals}

\section{Édition électronique}

URL : http://journals.openedition.org/conflits/18167

DOI : $10.4000 /$ conflits. 18167

ISSN : $1777-5345$

Éditeur :

CCLS - Centre d'études sur les conflits lilberté et sécurité, L'Harmattan

Édition imprimée

Date de publication : 30 décembre 2011

Pagination : 7-13

ISBN : 978-2-296-55700-0

ISSN : 1157-996X

\section{Référence électronique}

Delphine Lacombe, Elisabeth Marteu, Anna Jarry-Omarova et Brigitte Frotiée, « Le Genre globalisé : cadres d'actions et mobilisations en débats », Cultures \& Conflits [En ligne], 83 | Automne 2011, mis en ligne le 04 janvier 2013, consulté le 30 mars 2021. URL : http://journals.openedition.org/conflits/ 18167 ; DOI : https://doi.org/10.4000/conflits.18167 


\title{
Le Genre globalisé : cadres d'actions et mobilisations en débats
}

\author{
Introduction
}

\section{Delphine LACOMBE, Elisabeth MARTEU, Anna JARRY-OMAROVA, Brigitte FROTIEE}

$\mathrm{D}$ e la « décennie de la femme » après la conférence internationale de Mexico (1975), aux politiques de "gender mainstreaming » à la suite de la conférence de Pékin (1995), les luttes féministes ont connu une légitimation croissante sur la scène internationale. Dès lors, les politiques d'égalité femmes-hommes et la sexuation du langage des droits humains ont été portées aux échelles locales, nationales et internationales, avec pour point de mire les institutions étatiques et pour principal agent de promotion internationale l'Organisation des Nations Unies (ONU). La Convention sur l'élimination de toutes les formes de discriminations à l'égard des femmes (CEDAW) a ainsi été signée par 186 pays. Nouvelle terminologie d'usage pour désigner les rapports sociaux de sexe, le « genre » est devenu une catégorie globale d'intervention publique, dont l'intégration dans les politiques d'aide à destination des pays du Sud a été une dimension concrète majeure. Souvent présentés comme des instruments de luttes contre la pauvreté, ou encore comme outils de démocratisation et de pacification des États en sortie de conflits, divers projets de développement intégrant le genre ont été mis en œuvre par les gouvernements et/ou les acteurs de la «société civile ", relayant ainsi les prescriptions des institutions internationales. Les femmes en ont été des destinataires privilégiées, avec en particulier des activités dites « d'empowerment " (« empowerment économique » par les programmes de micro-crédits et «empowerment politique »), mais aussi avec des programmes de lutte contre les violences de genre ou pour la santé reproductive et sexuelle, ou enfin avec des projets de réforme du code de statut personnel dans certains pays du monde arabe et musulman. Ces activités ou programmes thématiques sont autant de déclinaisons d'interventions « de genre » soutenues par des bailleurs internationaux sous forme de projets de plus en plus standardisés et bureaucratisés.

Un débat tant scientifique que politique a émergé sur les modalités de ce processus. Les féminismes se divisent sur la question des contraintes et des 
ressources que présentent les projets de coopération « genre et développement ». Les voix les plus critiques constatent que la globalisation des « agendas de genre » a contribué à dépolitiser ce concept, en tentant d'uniformiser les pratiques. Dans le champ scientifique, l'indissociabilité de la globalisation économique et de l'internationalisation des programmes de développement a été soulignée. Les agents promoteurs de la « perspective de genre » ont pu être accusés tantôt de ne pas remettre profondément en cause le paradigme du néolibéralisme, tantôt d'y inscrire leurs schèmes d'actions en instrumentalisant le travail associatif féminin afin de pallier le renforcement des effets inégalitaires de la libéralisation économique. La massification et la féminisation de l'emploi précaire, l'accroissement du secteur informel, l'accentuation de la division sexuée du travail et des violences que subissent les femmes à l'échelle mondiale sont autant de constatations des universitaires et des militantes féministes locales.

Ce numéro de Cultures $\mathcal{E}$ Conflits s'inscrit au cœur de cette problématique en réinterrogeant le processus de globalisation du genre d'abord compris comme un changement d'échelle des enjeux politiques relatifs aux rapports sociaux de sexe. Dans les pas de Jean François Bayart, qui présente la globalisation notamment comme une « extension et intensification à l'échelle du monde des relations sociales ${ }^{1}$ », nous saisissons ici la façon dont les politiques de genre relèvent d'une programmatique portée par des institutions aux prétentions universelles et intégratives des enjeux sociaux d'espaces pourtant variables localement. Plus que l'analyse d'une uniformisation des politiques de genre et de l'automaticité de leur réception par les mobilisations et les gouvernements, il s'agit bien d'en saisir ici les effets négociés, contradictoires et conflictuels. En d'autres termes, si dans un premier temps nous avons pu nous appuyer sur l'hypothèse que la globalisation du genre était soit un facteur de dépossession soit un moyen de réappropriation de diverses ressources sociales, économiques et même politiques pour les femmes dans les sociétés où nous avons mené nos enquêtes, l'analyse des trajectoires et pratiques militantes réalisée dans le cadre de ce numéro ne se satisfait pas d'une explication aussi binaire ${ }^{2}$.

Quatre études de cas conduites en Inde, au Nicaragua, en Mongolie et en Israël/Palestine seront ainsi mises en perspective. Il s'agit ici de renseigner plus particulièrement la façon dont les associations locales de femmes développent leurs répertoires d'action sous l'effet de ressources et de contraintes à diffé-

1. Bayart J.F, Le gouvernement du monde. Une critique politique de la globalisation, Paris, Fayard, 2004.

2. L'ouvrage de Guérin I., Fraisse L., Hersent M. (eds), Femmes, économie et développement. De la résistance à la justice sociale, Paris, Eres, 2011, propose de la même façon de sortir d'une perspective dichotomique : «L'objectif premier de cet ouvrage consiste à prendre acte de ces formes d'action féminine [...]. Le deuxième objectif est d'ordre théorique : proposer une grille de lecture, en insistant sur leur dimension hybride et la nécessité de repenser nos catégories d'analyse. » (p. 9). 
rentes échelles tant locales et nationales qu'internationales. Ces contributions marquent ainsi leur originalité en cherchant à renseigner ces modes d'appropriation, leurs temporalités et les tensions inhérentes à l'articulation d'intérêts distincts (et souvent contradictoires) entre les associations, les États et les organisations internationales. Pourtant, si les contextes sociaux peuvent parâ̂tre différents, nous y observons une double dynamique similaire. D'une part, les associations locales/nationales de femmes, en vue d'obtenir les financements internationaux, se plient aux conditions normatives et matérielles imposées par les agences de développement. D’autre part, si le genre est « globalisé », ses enjeux restent bien souvent localisés. La singularité des sociétés réceptrices et les divers mouvements associatifs de femmes (y compris au sein d'un même pays) donnent à voir des adaptations et des réappropriations composites des prescriptions internationales de genre. Ceci nous invite à saisir in situ la place et le rôle de l'espace international dans les actions collectives des femmes, ainsi que les effets de ces mobilisations féminines sur les modes de sociabilités locales, sur les rapports sociaux de sexe, ainsi que sur le cours politique des États.

De fait, la thématique de la « globalisation du genre » a été principalement étudiée sous l'angle d'une part des politiques de développement et d'autre part des mobilisations féminines/féministes transnationales. En effet, le processus globalisant et normalisant de l'usage du « genre » comme cadre d'action et d'inflexion des politiques transnationales et nationales a été essentiellement analysé à l'aune des perspectives "genre et développement » dans les pas de l'étude fondatrice d'Ester Boserup ${ }^{3}$, et dans une perspective souvent critique de la globalisation néolibérale ${ }^{4}$, tout comme l'étude des programmes de micro-crédits ${ }^{5}$. La recherche féministe a aussi mis au jour l'évolution des terminologies et des outils pour l'inclusion des femmes dans les programmes de développement ${ }^{6}$, en mettant l'accent sur leurs aboutissements récents que sont le mainstreaming de genre et l'empowerment des femmes ${ }^{7}$. La standardisation a-politique des programmes de genre et de sa promotion par les ins-

3. Boserup E., La femme face au développement économique, Paris, PUF, 1983.

4. Wichterich C., La femme mondialisée, Paris, Éditions Solin, Actes Sud, 2000. Mestrum F., «La pauvreté multidimensionnelle. La dynamique sémantique dans le discours de la Banque mondiale ", Mots, Les langages du politique, 2008, 88, pp. 25-37. Bisilliat J. (ed.), Regards de femmes sur la globalisation : approches critiques, Paris, Karthala, 2003, pp. 153-170

5. Guérin I., Palier J., Prevost B., Femmes et microfinance. Espoirs et désillusions de l'expérience indienne, Paris, AUF Editions, 2009.

6. Moghadam V., "WID, WAD, GAD. Integration of Gender in Development", in Gender and Society, Working Paper 3 in series Gender and Development, Women's Studies Programme, Birzeit University, 1995, pp. 1-30. Locoh T., Labourie Racape A., Tichit C., Genre et développement : des pistes à suivre, Textes d'une rencontre scientifique à Paris, 11-12, Paris, Manuel du Ceped, 1996. Voir également le numéro spécial «Les mots du développement. Trajectoires et pouvoirs ", Revue Tiers Monde, 4, 2009, en particulier l'article de Verschuur C., "Quel genre ? Résistances et mésententes autour du mot "genre" dans le développement ", "Les mots du développement. Trajectoires et pouvoirs ", Revue Tiers Monde, 4, 2009, pp. 785-803.

7. Bisilliat J., Verschuur C., Le Genre : un outil nécessaire, introduction à une problématique, Paris, L'Harmattan, 2000. Voir aussi en particulier toute la première partie de l'ouvrage 
tances onusiennes et les agences de développement a également fait l'objet, dans différentes régions du monde, d'essais critiques soucieux de rendre visibles les résistances féministes aux phénomènes d'institutionnalisation, de professionnalisation de la militance ${ }^{8}$ et de la transformation croissante des mouvements en ONG 9. Depuis les années 1990 ont été développés des travaux sur le processus de globalisation des agendas de genre, en interrogeant le développement de ce que Charlotte Bunch nomme un « féminisme global ». Ils ont permis de renseigner les stratégies et les formes de l'action collective transnationale de plaidoyer ${ }^{10}$, notamment l'usage fait des organisations et conférences internationales ${ }^{11}$. La conférence de Pékin en 1995 a fait l'objet d'un intérêt particulier tant elle a suscité la participation des associations de femmes et des réformes législatives dans les pays du Sud ${ }^{12}$. Certaines études ont plus particulièrement éclairé l'influence des ONG dans la construction de politiques spécifiques, telle que la lutte contre les violences de genre et pour les droits reproductifs ${ }^{13}$. En France, les travaux novateurs de Mireille DelmasMarty ${ }^{14}$ sur l'internationalisation du droit renseignent les modes de construction de dispositifs normés à vocation globale. Par ailleurs, de nombreuses études de cas ont décrit les concrétisations locales de ces schèmes globaux d'action, l'hétérogénéité des acteurs en charge des politiques de genre, la tangibilité de leurs effets sur les politiques nationales et sur les répertoires de l'action collective. Dans la continuité des études féministes postcoloniales ${ }^{15}$, ces travaux ont réinterrogé l'action des associations locales de femmes et des féminismes locaux dans un contexte globalisé ${ }^{16}$. Les effets de la circulation transnationale de normes ou de pratiques ont également fait l'objet de plusieurs tra-

Guérin I., Fraisse L., Hersent M. (eds), Femmes, économie et développement. De la résistance à la justice sociale, Paris, Eres, 2011.

8. Hammami R., "NGOs: The Professionalization of Politics”, Race and Class, 37(2), 1995, pp. 51-63. Falquet J., De gré ou de force. Les femmes dans la mondialisation, Paris, La Dispute, le Genre du Monde, 2008.

9. Jad I., "The NGO-isation of Arab Women’s Movements”, Al Raida, vol.XX, n¹00, 2000, pp. 37-47. Alvarez S., “Advocating Feminism: The Latin American Feminist NGO 'Boom””, International Feminist Journal of Politics, vol. 1, Issue 2, August 1999, pp. 181-209.

10. Keck M.E., Sikkink K., Activists Beyond Borders: Advocacy Networks in International Politics, Cornell University Press, 1998.

11. Chen M. A., “Engendering World Conferences: The International Women's Movement and the UN", in Weiss T., Gordenker L. (eds), NGOs, the UN and Global Governance, London, Boulder, Lynne Rienner Publishers, 1996, pp. 139-158. Berkovitch N., From Motherhood to Citizenship: Women's Rights and International Organizations, Baltimore, Johns Hopkins University Press, 1999.

12. Afkhami M., Friedl E., Muslim Women and the Politics of Participation: Implementing the Beijing Platform, Syracuse University Press, 1997.

13. Jutta M. J., Agenda setting, the UN, and NGOs: Gender violence and reproductive rights, Georgetown University Press, 2007.

14. Delmas Marty M., Les forces imaginantes du droit: Tome 3, La refondation des pouvoirs, Paris, Le Seuil, 2007.

15. Voir récemment Verschuur C., "Genre, postcolonialisme et diversité des mouvements de femmes », Cabiers Genre et Développement, n7, 2010.

16. Basu A. (ed.), The Challenge of Local Feminisms: Women's Movements in Global Perspective, Boulder, Westview Press, 1995. Tawa Lama Rewal S., « Le local et le global dans le mouvement indien des femmes ", Cahiers du Genre, n40/2006, "Travail et mondialisation. Confrontations Nord/Sud », 2006, pp. 183-202. 
vaux, qu'il s'agisse du «voyage du genre 17», ou de problématiques non genrées, comme le développement ${ }^{18}$, l'humanitaire ${ }^{19}$ et la démocratie et les droits de l'humain 20.

Les perspectives de recherche présentées ici s’inscrivent dans la continuité de ces travaux tout en marquant leur singularité par un double décloisonnement, d'une part un décloisonnement des aires géographiques permettant la mise en évidence de processus locaux singuliers, d'autre part un décloisonnement par une analyse ciblée des modalités d'interrelations entre trois types d'acteurs : associations/États/bailleurs internationaux. Il s'agit dans ce numéro de questionner la circulation du genre afin d'aller au-delà d'un simple constat critique sur l'imposition de normes de genre vidées de leur sens subversif sous l'effet de la mondialisation néolibérale. Nous proposons d'analyser la manière dont les associations de femmes s'approprient les termes de ce débat à différentes échelles.

Ce faisant, chacune des recherches présentées ici s'articule autour d'une hypothèse centrale : si les dispositifs d'internationalisation du genre ont des effets structurants et créent indéniablement des dépendances financières et politiques, des effets de recompositions, de réappropriations et de résistances sont aussi à l'œuvre. Les rapports entre les associations locales de femmes et les acteurs internationaux ne sont pas unilatéraux. En centrant l'attention sur les expériences collectives et individuelles au sein des associations de femmes, sur les carrières militantes ou la reconfiguration des mobilisations, mais aussi sur les temporalités des revendications et des politiques genrées, nos analyses consistent autant à dévoiler les mécanismes de co-construction de ces dispositifs que révéler les stratégies d'adaptation ou d'évitement de la part des militantes locales.

Les quatre articles présentés mettent l'accent sur la façon dont des femmes, inscrites ou non dans des actions collectives, agissent sous l'effet de la diffusion de ressources discursives et matérielles de programmes censés leur venir en aide. Les articles saisissent aussi, chacun à leur manière, les marges de manœuvre des femmes dans leurs rapports de force avec les institutions étatiques et les représentants des organisations internationales ou des bailleurs de

17. Marques Pereira B., Meier P., Paternotte D., Au-delà et en deçà de l'État. Le genre entre dynamiques transnationales et multi-niveaux, Louvain-la-Neuve, Academia Bruylant, 2010.

18. Deler J.-P.., Faure YA., Piveteau A., Roca PJ., ONG et développement : société, économie et politique, Paris, Karthala, 1998. Hours B., Selim M., Anthropologie politique de la globalisation, Paris, L'Harmattan, coll. « Anthropologie critique », 2010.

19. Siméant J., «Entrer, rester en humanitaire », Dossier spécial «Devenir militants », Revue française de science politique, vol.51, n¹-2, février-avril 2001, pp. 47-72.

20. Dezalay Y., Garth B., "Droits de l'homme et philanthropie hégémonique », Actes de la recherche en sciences sociales, 121-122, pp. 23-41. Guilhot, N., The Democracy Makers: Human Rights and the Politics of Global Order, New York, Columbia University Press, 2005. Poujol C., "Perception et traitement de l'aide internationale en Asie Centrale depuis 1991 : trajectoire d'une suspicion », Hérodote, n¹29, 2008, pp. 21-35. 
fonds. Cependant, les situations d'arbitrage ou de conciliation continue entre agendas propres aux associations de femmes et adaptations stratégiques à des ressources extérieures soumises à conditions sont sources de tensions. Certaines associations de femmes sont parfois désignées comme étant un véhicule de l'hégémonie idéologique et culturelle des pays dominants, qui les conduiraient à dépolitiser les causes ou à pratiquer un militantisme élitiste. L'articulation entre féminisme et nationalisme, entre lutte contre le patriarcat et protection de la cellule familiale sont des exemples de points conflictuels entre des priorités parfois distinctes. Il en résulte également une diversité des formes de mobilisation et des définitions mêmes du féminisme.

L'article de Delphine Lacombe revient sur le débat autour de l'ONGisation de la militance féminine en Amérique Latine. En prenant appui sur l'expérience nicaraguayenne de recomposition des mobilisations de femmes après la révolution sandiniste, elle montre les tensions et le continuum entre adaptabilité stratégique et politiques de résistance féministes d'actions collectives hybrides (entre mouvement et ONG prestataires de services) où les militantes les plus influentes agissent depuis des positionnements multiples sphère gouvernementale, réseaux d'expertise, $O N G$, mouvements sociaux. Dans ce contexte, les débats de la militance féministe sur les contraintes et les ressources externes de leur action collective interviennent en contrepoint des enjeux de dépassement des impasses nationales relatives aux droits des femmes et à la construction démocratique. L'article d'Isabelle Guérin s'appuie sur une sociologie de la réception du micro-crédit en Inde pour saisir les modes d'appropriation des programmes d'aide tout en soulignant la permanence des pesanteurs sociales concernant les rapports sociaux de sexe, comme la non remise en cause de la division sexuée du travail en dépit de recompositions sociales à l'œuvre. L'article analyse les " capacités des femmes " non pas comme effet des programmes internationaux d'empowerment et de microfinance, mais comme l'une des conditions pour pouvoir en contourner, en réalité, les normes et les objectifs. L'auteur contribue, dans le sillage des travaux précurseurs de Francine Mestrum 21, à définir l'empowerment et sa mise en pratique par les programmes de développement comme un processus limité par ses orientations individualistes. Or, favoriser «l'émancipation des femmes» ne peut être dissocié des dimensions relationnelles du genre comme catégorie de pouvoir, et ses orientations individualistes. Elisabeth Marteu propose une analyse des modes de reconstruction de catégories d'actions, telles l'empowerment, le genre et le féminisme dans un contexte israélo-palestinien où financer des associations de femmes est souvent présenté comme un «travail de civilisation » à l'initiative de l'Occident. L'auteur montre que dans ce contexte fortement politisé et conflictuel, l'usage de l'épistémologie féministe par les associations de femmes révèle des problématiques identitaires comme

21. Voir entre autres Mestrum F., "L'“empowerment", le pouvoir et la capacité d'action ", Chronique féministe, 2000, pp. 70-75. 
des arbitrages stratégiques entre empowerment individuel et émancipation collective dans le cadre de mobilisations politiques. Enfin, les tensions entre politiques étatiques, coopération internationale et mobilisations de femmes sont également mises à jour dans l'article d'Anna Jarry-Omarova, qui étudie l'échec du mouvement associatif des femmes en Mongolie depuis la démocratisation du pays. En s'appuyant sur les travaux de Keck et Sikkink, Anna Jarry-Omarova montre que le passage par les instances internationales pour faire valoir des droits et tenter de faire pression sur les leaders politiques à l'échelle nationale, n'est pas seulement producteur d'un «effet-boomerang » positif pour les femmes. Les obstacles dressés par les États et les gouvernements peuvent au contraire se voir renforcés et générer un « contre effet-boomerang " préjudiciable aux luttes pour la représentation politique des femmes. 